\title{
COMPARATIVE STUDIES OF LOW-INTENSITY SHORT-LENGTH ARCS
}

\author{
M. BAeva ${ }^{a, *}$, E. Siewert ${ }^{b}$, D. Uhrlandt ${ }^{a}$ \\ ${ }^{a}$ Leibniz Institute for Plasma Science and Technology, Felix-Hausdorff-Strasse 2, 17489 Greifswald, Germany \\ ${ }^{b}$ Linde AG, Geschäftsbereich Linde Gas, Carl-von-Linde-Strasse 25, 85716 Unterschleissheim, Germany \\ * baeva@inp-greifswald.de
}

\begin{abstract}
We present results obtained by two non-equilibrium modelling approaches and experiments on low-intensity short-length arcs in argon at atmospheric pressure. The first one considers a quasineutral arc column combined with boundary conditions on the electrodes based on the energy balance in the space-charge sheaths. The second approach applies a unified description over the entire gap and solves the Poisson equation for the self-consistent electric field. The experiments provide the arc voltage.
\end{abstract}

Keywords: non-equilibrium, unified, arc plasma, modelling, experiment.

\section{Introduction}

Electric arcs of short length operated at a current level of several Amperes are not well understood, neither from an experimental nor from a modelling point of view. Most of the reported measurements on electric arcs were made for arc lengths of $3 \mathrm{~mm}$ and more. Lowcurrent electric arcs depart presumably from Local Thermodynamic Equilibrium (LTE) across the entire inter-electrode-region so that a non-equilibrium description is required. A self-consistent non-equilibrium modelling of tungsten-inert gas arcs of lengths down to $2 \mathrm{~mm}$ accomplished by experimental work have been reported in a recent authors' publication [1]. Some modelling studies on electric arcs of lengths $1 \mathrm{~mm}$ and below have been published in $[2,3]$. These studies have shown the transition from glow to arc in argon at atmospheric pressure. However, the heating of the tungsten cathode resulting from the arc-cathode interaction has not been done in a self-consistent manner, since a prescribed value of the cathode fall equal to the energy of ionization has been used. In recent publications $[4,5]$ by two of the authors of the present work, a unified one-dimensional and a self-consistent modelling of microdischarges and microarcs (interelectrode distance less than $1 \mathrm{~mm}$ ) has been presented and the properties of the microarc plasma have been analyzed. The results are found to be in a fairy qualitative agreement with experimental studies reported by Park et al [6]. Detailed experimental studies on microarcs, which gain importance in micro-welding and manufacturing, have not been reported yet.

In this work, we present the first results of comparative studies on short and low current arcs as shown by the organisation chart in Fig. 1. Two non-equilibrium modelling approaches and electrical measurements are employed. The paper is organized as follows. A brief description of the modelling approaches and the experiments is given in Section 2. Results and discussion are given in Section 3. Concluding remarks are summarized in Section 4.

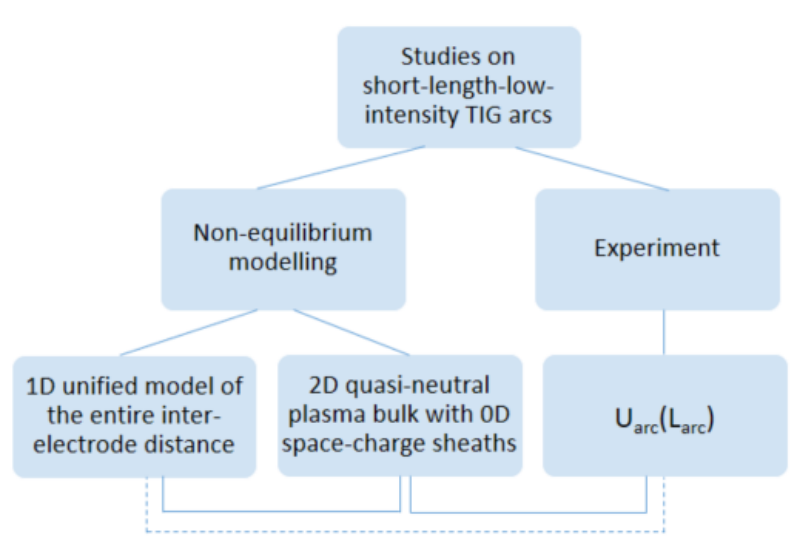

Figure 1. Organisation chart of the study.

\section{Methodology}

The studies comprise a non-equilibrium and selfconsistent modelling of the arc plasma and the electrodes, and electrical measurements in a tungsteninert gas configuration. The cathode is made of ceriated tungsten (WC20) and the anode is a water-cooled plate made of copper. The arc is burning in argon at atmospheric pressure. The inter-electrode distance, $L_{\text {arc }}$, is varied both in the experiment and in the models. The cathode is considered in the models with a length of $20 \mathrm{~mm}$.

\subsection{Modelling approaches}

Two modelling approaches are employed in the present study (see Fig. 1). They are referred to in the following as the 2D-fully-non-equlibrium model (2D-FNEM) and the 1D-unified-non-equilibriummodel (1D-UNEM). The detailed descriptions of the models are provided in $[4,5,7]$, respectively. Here, we outline briefly their common and specific features. Both models describe the plasma as a fluid that contains electrons and neutral and charged species of argon. The electrons are assumed to obey a Maxwellian velocity distribution and are characterized 
by the electron temperature $T_{\mathrm{e}}$, while the heavy particles are characterized by the common temperature $T$. Both models avoid assumptions of thermal and chemical equilibrium. Therefore, individual temperatures $\left(T_{\mathrm{e}} \neq T_{\mathrm{h}}\right)$ are related to the electrons and the heavy particles, and the plasma composition results from plasma-chemical reactions. Both models imply the bidirectional interaction between the arc plasma and the electrodes, accounting for fluxes between the plasma and the electrodes in both directions. In particular, the cathode is of central importance in tungsten-inert gas arcs, since the arc plasma is sustained by the thermionic emission of electrons from the cathode.

The 2D-FNEM is based on the magnetohydrodynamic description of the axially symmetric quasi-neutral plasma bulk. Hence, the Navier-Stokes equations of conservation of mass, momentum, energy of electrons and heavy species, and the conservation of ion species are solved along with the equation of current continuity, the Maxwell equations for the magnetic vector potential, the generalized Ohm's law, and the Dalton's law. The model further solves the heat and current transfer in the solid bodies of the electrodes. The 2D-FNEM employs the Stefan-Maxwell equations and the effective binary diffusion approach in a multi-component plasma, which is composed of electrons, singly charged argon ions and ground state atoms, to build a general diffusion formulation. This formulation gives rise to the generalized Ohm's law, in which the electric current density contains a drift component (proportional to the electric field) and a diffusion component (accounting for gradients of the mass fractions of species, the pressure, the temperatures). The space-charge sheath with a spatial extent of the order of the Debye length adjacent to the electrodes are not spatially resolved. Instead, their properties are accounted for in the boundary conditions on the boundary between the plasma and the electrodes. That is, the integral energy balance of electron energy and the integral balance of total energy in the space-charge sheath are important since they provide comprehensive boundary conditions.

The 1D-UNEM is aimed at solving the equations of particle and energy conservation for electrons and heavy species, the Poisson equation for the electric potential, the equation of heat-transfer in the cathode, and an electric circuit equation. The entire inter-electrode distance is spatially resolved, i.e. the quasi-neutral plasma bulk and the regions of space-charge are considered together. The 1D-UNEM employs the drift-diffusion approximation for the electron particle and energy flux and allows us therefore to account for the drift and diffusion components of the electric current density. Since low current arcs are of interest, the ionization degree of the generated plasma is assumed to be low enough so that the magnetic field and magnetohydrodynamic effects are ignored. A convective motion of the plasma and a self-induced magnetic field are not included in the model. The plasma bulk and the near-electrode regions are characterized by very different scales. Therefore, the spatial resolution in the unified model sets an important limit concerning the computational overhead even in 2D treatment. The unified model is so far realized in 1D and its applicability is therefore restricted to plane-parallel configurations which length is much shorter than their radial extent.

\subsection{Electrical measurements}

The detailed description of the experimental setup and the evaluation of the results covering the electrical measurements can be found in [1]. The measurements are aimed at providing the arc voltage $U_{\text {arc }}$ for various arc lengths $L_{\text {arc }}$ in a tungsten-inert gas configuration. The cathode is a cylindrical rod with a radius of $2 \mathrm{~mm}$, and a conical tip with an angle of $30^{\circ}$ and a plateau radius of $0.15 \mathrm{~mm}$. An arc current of $5.3 \mathrm{~A}$ is considered in the present study. The arc voltage is measured for arc lengths starting from $2 \mathrm{~mm}$ in $0.025 \mathrm{~mm}$ steps until a short circuit occurs. The zerovalue of $L_{\text {arc }}$ is determined by the position of the short circuit.

\section{Results and discussion}

The 2D-FNEM is applicable to an arbitrary axially symmetric tungsten-inert gas configuration. In order to enable a direct comparison with the experiment, the experimental setup with a conical tip of the cathode is reproduced in the model. The 1D-UNEM is inapplicable to this configuration due to the small radius of the cathode tip. Nevertheless, a comparison of the results between the 1D-UNEM and the 2D-FNEM can be done for a configuration with a cylindrical cathode rod with a radius $R=2 \mathrm{~mm}$ and $L_{\text {arc }} \leq 1 \mathrm{~mm}$, for which the one-dimensional treatment is still justified. Experimental data for a configuration with a flat cathode is still not available. Therefore, the comparisons model-to-experiment and model-to-model as indicated in Fig. 1 can be done. The objective includes two important tasks that are new. First, the comparison 2D-FNEM to experiment will prove the predictive capability of the 2D-FNEM at very short arc lengths and low currents. Second, the comparison $1 \mathrm{D}-\mathrm{UNEM}$ to $2 \mathrm{D}-\mathrm{FNEM}$ will prove the accuracy level of the modelling approach based on the unresolved space-charge sheath and its average properties at the conditions under consideration.

\subsection{D-FNEM and experiment}

Figure 2 shows an image of the arc plasma and the calculated two-dimensional distribution of the temperature for an inter-electrode distance of $0.4 \mathrm{~mm}$. The CCD image illustrates the plasma cloud around the cathode, which tip end is hidden by the plasma but still visible. The calculated distribution is taken in 

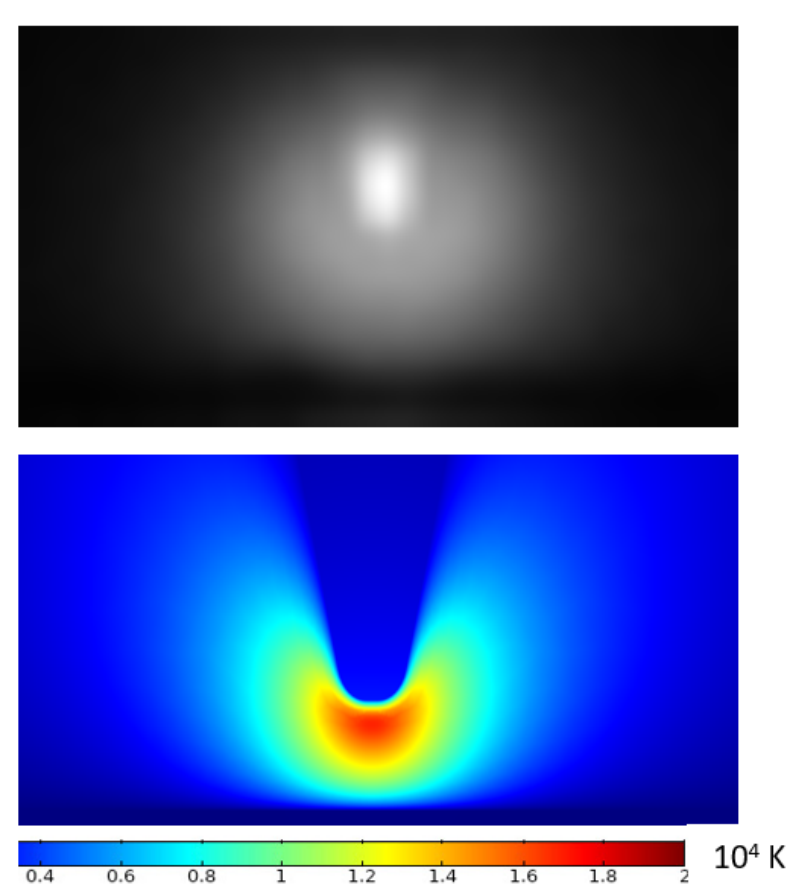

Figure 2. CCD-image of the arc and the distribution of the gas temperature of the arc at $L_{\text {arc }}=0.4 \mathrm{~mm}$ from the 2D-FNEM.

the plane through the axis of symmetry and shows the end of the cathode tip. At the conditions under consideration $\left(L_{\mathrm{arc}}=0.4 \mathrm{~mm}, I_{\mathrm{arc}}=5.3 \mathrm{~A}\right)$, the arc shape differs from the well known bell shape of high-current arcs. The magneto-hydrodynamic simulation shows that flow jet generated by the Lorentz force is of minor importance. The arc plasma spreads therefore in radial direction.

The short-length low-current arcs are in nonequilibrium (Fig. 3). The thermal non-equilibrium is stronger pronounced in the regions in front of the electrodes than in the middle of the plasma column. For shorter arc lengths, $T_{\mathrm{e}}$ remains higher close to the anode, while close to the cathode it approaches almost the same value of about $25000 \mathrm{~K}$. The departure between $T$ and $T_{\mathrm{e}}$ is less pronounced in the middle of the arc for a longer $L_{\text {arc }}$.

Figure 4 presents the arc voltage $U_{\text {arc }}$ obtained in the experiment and the calculated one for arc lengths below $2 \mathrm{~mm}$. The experimental values are obtained in two series and show a difference between 1 and $2 \mathrm{~V}$ due to the less stability of the arc at low current density. The values at $L_{\text {arc }} \rightarrow 0$ indicate the total voltage drop in the space-charge sheaths. The calculated arc voltage values lie well within the experimental span. The arc voltage in the model is the resultant of the voltage drops in the space-charge sheaths, the arc column $\left(U_{\mathrm{pl}}\right)$ and the solid bodies. Since the voltage drop in solids is in the order of $0.1 \mathrm{~V}$ and $U_{\mathrm{pl}}$ is below $2 \mathrm{~V}$ in the range of arc lengths considered, the arc voltage contains most the voltage drop in the space-charge sheaths, in particular in the

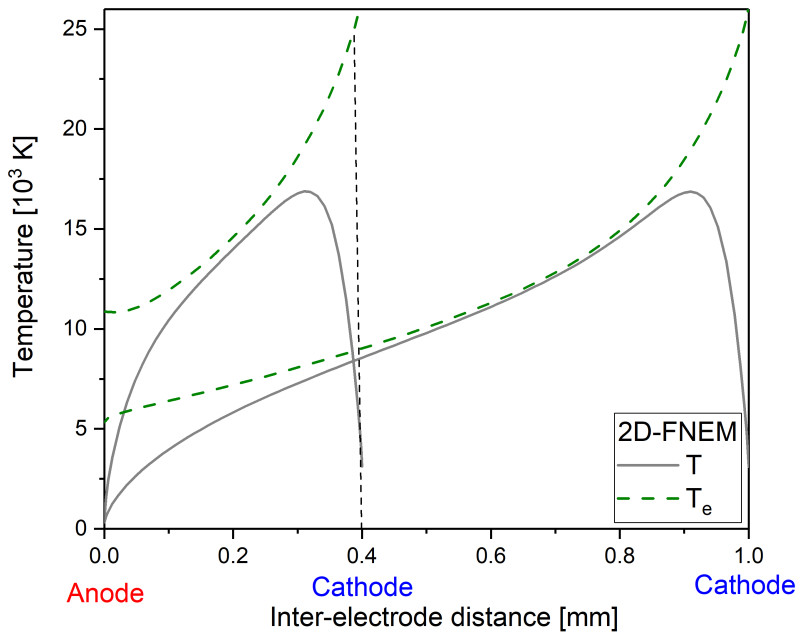

Figure 3. Temperatures of electrons and heavy particles at an electric current of $5.3 \mathrm{~A}$ from the $2 D-F N E M$ for arc lengths of 0.4 and $1 \mathrm{~mm}$.

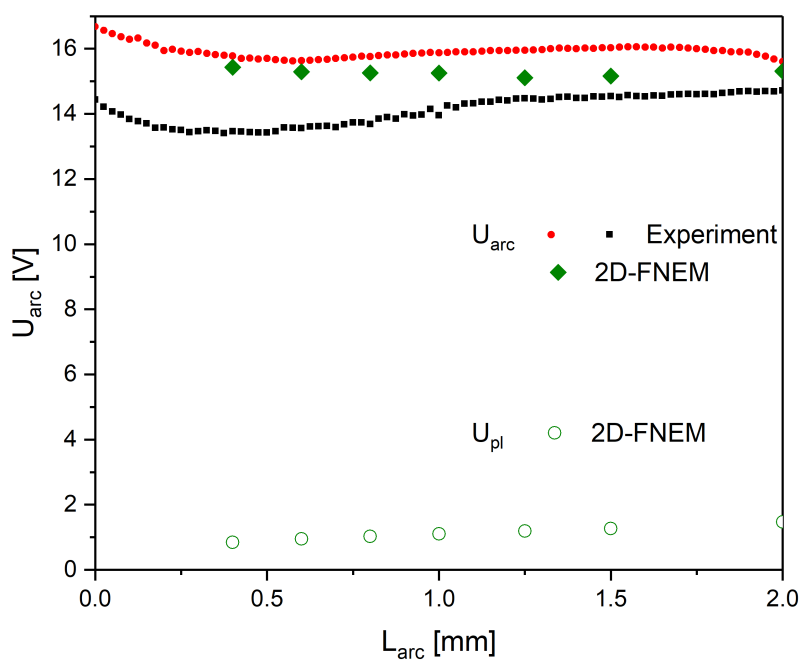

Figure 4. The arc voltage as a function of the arc length at an electric current of $5.3 \mathrm{~A}$ from the experiment and the 2D-FNEM.

cathode sheath of the tungsten-inert gas arc. The good agreement between the experimental and the modelling results is not trivial. We have shown in [1] that the 2D-FNEM is capable of predicting the arc plasma properties and in particular the arc voltage over the range of arc currents (5-200) A and arc lengths $(2-20) \mathrm{mm}$. For these $L_{\text {arc }}$ values, the axial extent of the arc is much longer than the spatial extent of the space-charge sheaths, which are unresolved in the model but are considered as zero-dimensional. This inequality becomes less justified for arc lengths in the sub-millimeter range. Below, we consider the spatial structure of short-length arcs.

\subsection{D-UNEM and 2D-FNEM}

Figure 5 shows the spatial distribution of the electron and ion density, and the electric potential obtained by the 1D-UNEM for the shortest arc length considered 


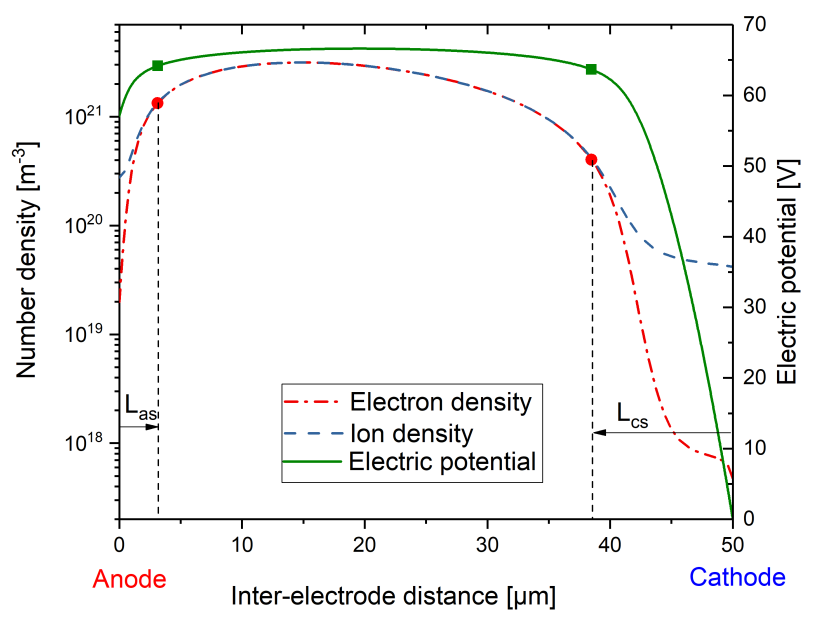

Figure 5. The spatial distribution of the electron and ion density and the electric potential from the $1 D$ UNEM at an electric current of $5.3 \mathrm{~A}$.

in the study $\left(L_{\mathrm{arc}}=0.05 \mathrm{~mm}\right)$ and an arc current of 5.3 A. The edge of the regions of quasi-neutrality and the space-charge sheaths adjacent to the electrodes are indicated by the symbols, which correspond to the position, where the ion density amounts $1 \%$ of the difference between the ion and electron density. The distances $L_{\mathrm{as}} \simeq 3 \mu \mathrm{m}$ and $L_{\mathrm{cs}} \simeq 13 \mu \mathrm{m}$ are then the estimates of the lengths of, respectively, the anode and the cathode space-charge sheath. With the decrease of the arc length, the length of the arc column tends to vanish and the anode and the cathode sheaths merge. The 2D-FNEM becomes inapplicable at such conditions. From Figure 5, one can obtain the voltage drops in the anode and the cathode sheaths. The voltage drop in the anode sheath is negative and amounts about $-7.1 \mathrm{~V}$ while the voltage drop in the cathode sheath is about $64.4 \mathrm{~V}$ and exceeds the total arc voltage of about $57 \mathrm{~V}$.

The arc voltage decreases with the increase of the arc length as shown in Fig. 6. The increase of the arc voltage at vanishing arc length is also observed experimentally and predicted by the 2D-FNEM (Fig. 4). Results from the 2D-FNEM for arc lengths below $2 \mathrm{~mm}$ and a cylindrically shaped cathode, which can be directly compared with those of the 1D-UNEM, are still forthcoming. For an arc length of $5 \mathrm{~mm}$, the 2D-FNEM predicts an arc voltage of $27.6 \mathrm{~V}$.

\section{Conclusions}

The comparative studies presented here show that the 2D-FNEM is a powerful tool for modelling of short-length low-current tungsten-inert gas arcs. The modelling results agree well with electric measurements. The 1D-UNEM delivers important knowledge about the spatial structure of the arc plasma and the plasma parameters in the space-charge sheaths. It can be applied in cases of very short arc lengths, for which the 2D-FNEM becomes inapplicable. Future

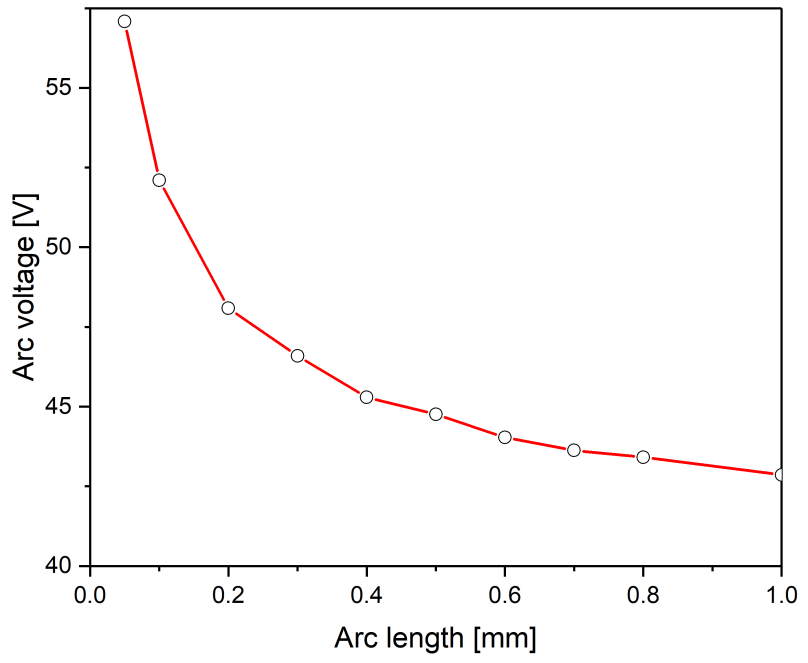

Figure 6. The arc length-voltage characteristics from the 1D-UNEM at an electric current of $5.3 \mathrm{~A}$.

works will focus on the analysis of the average plasma properties in the sheaths employed in the 2D-FNEM and spatially resolved ones in the 1D-UNEM.

\section{Acknowledgements}

The work of M. Baeva is funded by the Deutsche Forschungsgemeinschaft (DFG, German Research Foundation) - Project number 390828847.

\section{References}

[1] E. Siewert, M. Baeva, and D. Uhrlandt. The electric field and voltage of dc tungsten-inert gas arcs and their role in the bidirectional plasma-electrode interaction. $J$. Phys. D: Appl. Phys. (under review), 2019.

[2] S. I. Eliseev, A. A. Kudryavtsev, H. Liu, Z. Ning, and A. S. Chirtsov. Transition from glow microdischarge to arc discharge with thermionic cathode in argon at atmospheric pressure. IEEE Trans. Plasma Sci., 44:2536-2544, 2016. doi:10.1109/TPS . 2016. 2557587.

[3] A. I. Saifutdinov, I. I. Fairushin, and N. Kashapov. Analysis of various scenarios of the behavior of voltage-current characteristics of direct-current microdischarge at atmospheric pressure. JETP Lett., 104:180-185, 2016. doi:10.1134/S0021364016150145.

[4] M. Baeva, D. Loffhagen, M. M. Becker, and D. Uhrlandt. Fluid modelling of DC argon microplasmas: effects of the electron transport description. Plasma Chem. Plasma Process., 39:949-968, 2019. doi:10.1007/s11090-019-09994-5.

[5] M. Baeva, D. Loffhagen, and D. Uhrlandt. Unified non-equilibrium modelling of tungsten-inert gas microarcs in atmospheric pressure argon. Plasma Chem. Plasma Process., 2019.

[6] M. Park, Y. Hirata, and T. Urabe. Development of controlled micro-discharge at the atmospheric pressure. Weld. World, 58:47-54, 2014. doi:10.1007/s40194-013-0092-9.

[7] M. Baeva, M. S. Benilov, N. A. Almeida, and D. Uhrlandt. Novel non-equilibrium modelling of a DC electric arc in argon. J. Phys. D: Appl. Phys., 49:245205, 2016. doi: 10 .1088/0022-3727/49/24/245205/meta. 